Pacific Journal of Mathematics

EXTENSIONS OF SUBADDITIVE FUNCTIONS 


\title{
EXTENSIONS OF SUBADDITIVE FUNCTIONS
}

\author{
RICHARD G. LAATSCH
}

1. Introduction. A function $f$ defined on a set $H$ of real numbers is subadditive on $H$ if $f(x+y) \leqq f(x)+f(y)$ for all $x, y \in H$ such that $x+y \in H$. If the inequality is reversed, $f$ is superadditive. This paper considers several problems in the extension of subadditive and monotone subadditive functions to domains of which the given $H$ is a proper subset, and some related problems. First, extensions of a function from the set $J$ of all nonnegative integers of the set $E=[0, \infty)$ will be discussed. Then extensions of functions from $E$ to the set of all real numbers and extensions from an interval $[0, a]$ to $[0, b](b>a)$ and to $E$ will be discussed. This last discussion will emphasize the maximal extension first defined by Bruckner [1] in the superadditive case, will treat the problem of convergence of sequences of such extensions, and will study the operator properties of the extension. Finally, an example will be considered which is relevant to a problem in extremal elements of cones of functions.

2. Extensions from the integers. Let $f$ be a subadditive function defined on $J$. It has been shown that the polygonal extension, $F$, of $f$ to $E$, obtained by joining consecutive points $(n, f(n))$ of the graph of $f$ by straight line segments, is subadditive on $E$. [1]. It is easy to show that the left-continuous step function extension $G$, defined by $G(0)=f(0)$ and $G(x)=f(n)$ for all $x \in(n-1, n](n=1,2, \cdots)$ is subadditive on $E$ when $f$ is nondecreasing. These two extensions appear as the extreme cases of the class of extensions described in the following result.

THEOREM 1. Let $f$ be a nondecreasing subadditive function on J. Let $g$ be a nondecreasing concave function on $[0,1]$ with $g(0)=0$ and $g(1)=1$. The function $F$ defined on $E$ by

$$
F(x)=f([x])+\{f([x+1])-f([x])\} g(x-[x]),
$$

where $[x]$ is the integer $x-1<[x] \leqq x$, is subadditive and nondecreasing on $E$.

Proof. The function $F$ is obviously nondecreasing. To show subadditivity, let $x=m+u$ and $y=n+v$, where $m, n \in J$ and

Received May 1962, and in revised form August 1962. This paper is a part of the author's doctoral thesis, written at Oklahoma State University under the direction of Professor E. K. McLachlan. 
$u, v \in[0,1)$. If $u+v=h \leqq 1$ and $g(u)+g(v) \leqq 1$, then $g$ is subadditive on $[0,1]$ since $g(x) / x$ is nonincreasing there, and

$$
\begin{aligned}
F(x+y) \leqq & f(m+n)+\{f(m+n+1)-f(m+n)\}\{g(u)+g(v)\} \\
\leqq & \{f(m)+f(n)\}\{1-g(u)-g(v)\} \\
& +\{f(m+1)+f(n)\} g(u)+\{f(m)+f(n+1)\} g(v) \\
\leqq & f(m)+\{f(m+1)-f(m)\} g(u)+f(n) \\
& +\{f(n+1)-f(n)\} g(v)=F(x)+F(y) .
\end{aligned}
$$

If $g(u)+g(v)>1$, assume notation such that $f(n+1)-f(n) \leqq$ $f(m+1)-f(m)$. Then $\{f(n+1)-f(n)\}\{g(u)+g(v)\}+f(n) \geqq f(n+1)$, and it follows that

$$
\begin{aligned}
F(x+y) \leqq & f(m+n+1) \leqq f(m) \\
& +\{f(n+1)-f(n)\}\{g(u)+g(v)\}+f(n) \\
\leqq & f(m)+\{f(n+1)-f(n)\} g(u)+f(n) \\
& +\{f(n+1)-f(n)\} g(v) \leqq F(x)+F(y) .
\end{aligned}
$$

If $u+v=1+h>1$, the concavity of $g$ yields the inequality $g(h) \leqq$ $g(u)+g(v)-1$. Then

$$
\begin{aligned}
F(x+y) \leqq & f(m+n+1) \\
& +\{f(m+n+2)-f(m+n+1)\}\{g(u)+g(v)-1\} \\
\leqq & \{f(m)+f(n+1)\}\{1-g(u)\}+\{f(m+1)+f(n)\}\{1-g(v)\} \\
& +\{f(m+1)+f(n+1)\}\{g(u)+g(v)-1\}=F(x)+F(y) .
\end{aligned}
$$

Simple examples show that the theorem fails if either hypothesis on $f$ or $g$ is removed.

A similar but much simpler proof can be given for the following construction of periodic subadditive functions, which is suggested by the subadditivity of $|\sin x|$.

THEOREM 2. Let $g$ be concave and nonnegative on $[0,1)$. The extension, $F$, of $g$ to $E$ defined by $F(x)=g(x-[x])$ is subadditive on $E$.

The concavity of $g$ is not necessary, even if $g(0)=g(1)=0$, since the polygonal extension of the function defined on $\{0,1,2,3,4\}$ by $f(0)=f(4)=0, f(1)=f(3)=2$, and $f(2)=1$ can be extended to $E$ as a periodic subadditive function.

3. Extensions from $E$ to $R$. It is the purpose of this section to mention some results on the extension of a subadditive function $f$, defined on $E=[0, \infty)$, to the whole line $R$. An idea of what not to 
expect is provided by theorems of Hille and Phillips [2], who show that a finite-valued subadditive function defined on $(0, \infty)$ has no finite subadditive extension to $R$ if either $f(x) \rightarrow \infty$ as $x \rightarrow 0$ or if $f(x) / x \rightarrow-\infty$ as $x \rightarrow \infty$, and of Cooper [3], who has noted that every even $(f(x)=$ $f(-x)$ ) subadditive function is nowhere negative. The following theorem completely characterizes even subadditive functions.

THEOREM 3. Let $f$ be subadditive on $E$. Then $f$ can be extended to a subadditive even function on $R$ if, and only if, $f(x-y) \leqq$ $f(x)+f(y)$ for all $x \geqq y$ in $E$.

THEOREM 4. If $f$ is nondecreasing and subadditive on $E$ and nonincreasing and subadditive on $(-\infty, 0)$, then $f$ is subadditive on $R$.

CoRollary. Every nondecreasing subadditive function defined on $E$ can be extended to $R$ as an even subadditive function. Every nondecreasing subadditive function $f$ on $E$ can be extended as a subadditive function to $R$ by $f(x)=0$, or by $f(x)=f(0)$, for all $x<0$.

On the other hand, a nonincreasing subadditive function on $E$ can be extended to $R$ as an even subadditive function if, and only if, $\sup \{f(x): x \in E\} \leqq 2(\inf \{f(x): x \in E\})$.

4. Extensions from $[0, a]$ to $E$. The following discussion concerns the extension of a subadditive function $f$ defined on the interval $[0, a]$, $a>0$, to $E=[0, \infty)$ or to an interval $[0, b], b>a$. The inclusion of the origin is sometimes convenient and often a nuisance. There is an obviously parallel theory for extensions from $J_{k}=\{0,1,2, \cdots, k\}$ to $J$ which, together with Bruckner's theorem on polygonal extensions, provides a fruitful collection of examples in the continuous case. Two simple ways of extending monotone functions will be mentioned first.

THEOREM 5. Let $f$ be a nondecreasing subadditive function on $[0, a]$. Extend $f$ by $F(x)=f(x)$ if $x \in[0, a]$ and by $F(x)=f(a)$ if $x>a$. Then $F$ is subadditive on $E$.

THEOREM 6. Let $f$ be nondecreasing and subadditive on $[0, a]$. Let $g$ be defined by $g(x)=f(x)$ if $x \in[0, a], g(x)=f(a)$ if $x \in(a, 2 a]$, and $g(x)=f(a)+f(x-2 a)$ if $x \in(2 a, 3 a]$. Then $g$ is subadditive .on $[0,3 a]$.

Proof. Note that $g$ is also nondecreasing (which means that this construction can be repeated as often as desired). If $x, y, x+y \in[0,2 a]$, then $g$ is subadditive by Theorem 5 . If $x+y \in(2 a, 3 a]$, then, by 
cases, (1) if $x, y \in(a, 2 a]$,

$$
g(x+y) \leqq g(3 a)=2 f(a)=g(x)+g(y) ;
$$

(2) if $x \in[0, a]$ and $y \in(a, 2 a]$, then $y-2 a \leqq 0$ and

$$
g(x+y)=f(a)+f(x+y-2 a) \leqq f(a)+f(x)=g(x)+g(y) ;
$$

and (3) if $x \in[0, a]$ and $y \in(2 a, 3 a]$, then

$$
\begin{aligned}
g(x+y) & =f(a)+f(x+y-2 a) \\
& \leqq f(a)+f(x)+f(y-2 a) \leqq g(x)+g(y) .
\end{aligned}
$$

Attention now turns to the topic of maximal extensions. Let $f$ be a subadditive function on the interval $[0, a]$. The function $S f$ defined at each $x \in E$ by $S f(x)=\inf \Sigma f\left(x_{i}\right)$, where the infimum is taken over all finite collections $\left\{x_{1}, \cdots, x_{n}\right\}$ such that $0 \leqq x_{i} \leqq a(i=$ $1, \cdots, n)$ and $x_{1}+\cdots+x_{n}=x$ is called the maximal subadditive extension of $f$ to $E$. Each collection $\left\{x_{1}, \cdots, x_{n}\right\}$ is called an a-partition of $x$. It is verifiable that $S f$ is subadditive and $S f(x) \geqq F(x)$ for all $x$ and all subadditive functions $F$ on $E$ which are extensions of $f$. For any given $a$-partition of $x$ it can be shown, using $f\left(x_{i}+x_{j}\right) \leqq$ $f\left(x_{i}\right)+f\left(x_{j}\right)$, that there exists an $a$-partition (called a refinement of the given one) which does not contain 0 and does contain at most one element $v \leqq a / 2$-providing an approximation to $S f(x)$ at least as good as the original with the additional feature of an upper bound on $n$. These ideas have been discussed by Bruckner [1] for the analogous case of minimal extensions of superadditive functions. Contrary to the spirit of that paper, assumptions of continuity are avoided in the following discussion.

THEOREM 7. Let $f$ be subadditive on $[0, a]$. Then $f$ is nondecreasing on $[0, a]$ if, and only if, $S f$ is nondecreasing on $E$.

Proof. Since $f$ is the restriction of $S f$ to $[0, a]$ (denoted hereafter " $f=S f \mid[0, a]$ "), the monotonicity of $f$ follows from that of $S f$. Conversely, if $S f$ decreases, then there exist $x, y \in E$ such that $0<y-x<a / 2$ and $S f(y)<S f(x)$. If $y \in[0, a]$, then the argument is complete. If $y>a$, let $\varepsilon>0$ be given. Let $\left\{y_{1}, \cdots, y_{n}\right\}$ be a refined $a$-partition of $y$ such that $y_{1}>a / 2$ and $S f(y)+\varepsilon>f\left(y_{1}\right)+\cdots+f\left(y_{n}\right)$. Let $z=y_{1}-(y-x)$. Then $\left\{z, y_{2}, \cdots, y_{n}\right\}$ is an $a$-partition of $x$, so that $S f(x) \leqq f(z)+f\left(y_{2}\right)+\cdots+f\left(y_{n}\right)$. Subtraction yields $S f(y)-S f(x)+\varepsilon>f\left(y_{1}\right)-f(z)$, implying that $f$ decreases on $[0, a]$. A slight amendment of this argument verifies that "strictly increasing" may be substituted for "nondecreasing" in the theorem. 
THEOREM 8. If $f$ is subadditive on $[0, a]$, if $0<c<a$, and if $g=f \mid[0, c]$, then $S g(x) \geqq S f(x)$ for all $x \in E$. Also, $S g=S f$ if, and only if, $S g \mid[0, a]=f$.

The somewhat tedious proof of this theorem is omitted. These two theorems have served to emphasize the regularity of behavior of $S f$. Treated as an operator on the set $T$ of all subadditive functions on $[0, a], S$ is a monotone, positive-homogeneous, superadditive operator, and is additive on certain subsets of T. In particular, $S(f+g)=$ $S f+S g$ if $g$ is a nonnegative scalar multiple of $f$, or if $f$ and $g$ are concave and nonnegative at 0 . The concave functions satisfy the condition of the following theorem, and it should be noted that the set of all functions satisfying the condition is closed under addition and that $S$ is additive on this set.

THEOREM 9. Let $f$ be a subadditive function on $[0, a]$ such that $S f(a+x)=f(a)+f(x)$ for all $x \in(0, a]$. Then $S f(m a+x)=$ $m f(a)+f(x)$ for all $m \in J$ and all $x \in(0, a]$.

The proof of this theorem involves generating an $a$-partition of $y \in(a, \infty)$ of the form $\{a, a, \cdots, a, x\}$ from an arbitrary $a$-partition and using the hypothesis to show that it yields $S f(m a+x)$. A similar method can be used to show that, if $S f((n+1) a+x)=f(a)+S f(n a+x)$ for some $n \in J$ and all $x \in(0, a]$, then $S f(m a+x)=(m-n) f(a)+S f(n a+x)$ for all $m \geqq n$ and all $x \in(0, a]$.

5. Boundedness and convergence of maximal extensions. The following theorem generalizes a result of Bruckner [1], who usually assumes continuity or differentiability.

THEOREM 10. If $f$ is a bounded subadditive function on $(0, a]$, if $m=\inf \{f(x) / x: x \in(0, a]\}$, and if $b=\sup \{f(x)-m x: x \in(0, a]\}$, then $m x \leqq S f(x) \leqq m x+b$ for all $x \in(0, \infty)$.

Proof. Since $f(2 x) \leqq 2 f(x)$ implies $f(2 x) / 2 x \leqq f(x) / x$ for $x \in(0, a / 2]$, only values of $x \in(a / 2, a]$ need to be considered in finding a lower bound of $f(x) / x$. Since $f$ is bounded, both $m$ and $b$ are easily shown to exist. Consider $\varepsilon>0$ and $y \in(a, \infty)$. Let $\left\{x_{1}, \cdots, x_{n}\right\}$ be a refined $a$-partition for $y$ such that $S f(y)+\varepsilon \geqq f\left(x_{1}\right)+\cdots+f\left(x_{n}\right)$. Since $m \leqq f\left(x_{i}\right) / x_{i}(i=1, \cdots, n), m \leqq\left(\Sigma f\left(x_{i}\right)\right) / \Sigma x_{i} \leqq(S f(y)+\varepsilon) / y$, or $m y \leqq$ $S f(y)+\varepsilon$. Since $\varepsilon$ is arbitrary, $m y \leqq S f(y)$.

There exists a unique integer $p$ such that $y=p a / 2+z, 0 \leqq$ $z<a / 2$. Let $t \in(a / 2, a]$ such that $f(t) / t<m+\varepsilon / p a$. Then the integer $r$ is determined such that $y=r t+z^{\prime}$, where $0 \leqq z^{\prime}<t$. Note that 
$r \leqq p$. By definition, $S f(y) \leqq r f(t)+f\left(z^{\prime}\right)$. Since $f(t)<t m+t \varepsilon / p a$ and $f\left(z^{\prime}\right) \leqq m z^{\prime}+b$,

$$
\begin{aligned}
S f(y)<r(t m & +t \varepsilon / p a)+m z^{\prime}+b \\
& =m\left(r t+z^{\prime}\right)+b+t r \varepsilon / p a \leqq m y+b+\varepsilon .
\end{aligned}
$$

Thus $S f(y) \leqq m y+b$.

The proof of Bruckner's Theorem 3 can be used to show that, if $\left\{f_{n}\right\}$ is a sequence of continuous subadditive functions converging uniformly to the function $f$ on $[0, a]$, then $\lim S f_{n}=S f$. (That $f$ is subadditive follows, even for pointwise convergence, from a result stated in [2].) His proof makes use of the monotonicity of nonnegative superadditive functions to establish the uniform convergence. The statement, " $f_{n}$ subadditive and $f_{n}-f$ imply $S f_{n} \rightarrow S f$," is false, even for continuous nonnegative functions. For example, if $f_{n}$ is the polygonal extension to $[0,1]$ of the function $g_{n}$ defined by $g_{n}\left(1 / 2^{n}\right)=$ $g_{n}\left(1-1 / 2^{n}\right)=1 / 2$ and $g_{n}\left(k / 2^{n}\right)=k / 2^{n} \quad\left(k=0,2,3, \cdots, 2^{n}-2,2^{n}\right)$, and if $f(x)=x$ on $[0,1]$, then $f_{n} \rightarrow f, S f(x)=x$ on $E$, but $S f_{n}$ is tending in the direction of $y=(x+1) / 2$ by Theorem 10. However, other conditions which imply $S f_{n} \rightarrow S f$ can be given.

THEOREM 11. If $\left\{f_{n}\right\}$ is a sequence of subadditive functions on $[0, a]$ converging to $f$ there, and if $f_{n} \geqq f$ for all $n$, then $S f_{n} \rightarrow S f$ on $E$.

It is also noteworthy that the usual kinds of conditions implying uniform convergence can be modified for sequences of subadditive functions. In fact, a "classical" example, $n x /\left(1+n^{2} x^{2}\right)$, of nonuniform convergence on $E$ provides an example of a sequence of subadditive functions pertinent to Theorems 11 and 12 .

THEOREM 12. Let $\left\{f_{n}\right\}$ be a sequence of subadditive functions (not necessarily continuous) converging to the continuous function $f$ on $[0, a]$ and such that there exists a real number $m \geqq 0$ such that $f_{n}(x) \leqq m x$ for all $n$ and all $x \in[0, a]$. Then the convergence $f_{n} \rightarrow f$ is uniform on $[0, a]$.

6. The Cantor function. Let $K$ be the function defined on the complement of the Cantor "middle-third" set in $[0,1]$ by $K(x)=1 / 2$ if $x \in(1 / 3,2 / 3), K(x)=1 / 4$ if $x \in(1 / 9,2 / 9), K(x)=3 / 4$ if $x \in(7 / 9,8 / 9)$, etc., and by the limit at points of the Cantor set. The function $K$ is a frequently-used example in connection with continuity properties. To show that $K$ is subadditive, let $K_{n}(x)=K(x)$ if $x$ is in an interval which has been deleted from $[0,1]$ at the $n$th stage in the formation 
of the Cantor set and extend $K_{n}$ polygonally to $[0,1]$ with $K_{n}(0)=0$ and $K_{n}(1)=1$. Theorem 6 may be applied to show that $K_{n}$ is subadditive on $[0,1]$. Since $\lim K_{n}=K, K$ is subadditive. This example helps to illuminate the unsolved problem of characterizing the extremal elements of the convex cone of all nondecreasing subadditive functions on $[0,1]$, for $K$ is extremal. The other known extremal elements are of much simpler character.

\section{REFERENCES}

1. Andrew Bruckner, Minimal superadditive extensions of superadditive functions, Pacific J. Math., 10 (1960), 1155-1162.

2. Einar Hille and R. S. Phillips, Functional analysis and semigroups, Amer. Math. Soc. Colloquium Publ., Vol. 31, Providence, 1957, Chapter 7.

3. R. Cooper, The converse of the Cauchy-Hölder inequality and the solutions of the inequality $g(x+y) \leqq g(x)+g(y)$, Proc. London Math. Soc., (2) 26 (1927), 415-432.

OKLAHOMA STATE UNIVERSitY 



\section{PACIFIC JOURNAL OF MATHEMATICS}

\section{EDITORS}

Robert Osserman

Stanford University

Stanford, California

M. G. Arsove

University of Washington

Seattle 5 , Washington
J. DugundjI

University of Southern Califorma: Los Angeles 7, California

Lowell J. Paige

University of California

Los Angeles 24, California

\section{ASSOCIATE EDITORS}

E. F. BECKENBACH

B. H. NeumanN

F. WOLF

K. YosIDA

\section{SUPPORTING INSTITUTIONS}

UNIVERSITY OF BRITISH COLUMBIA

CALIFORNIA INSTITUTE OF TECHNOLOGY

UNIVERSITY OF CALIFORNIA

MONTANA STATE UNIVERSITY

UNIVERSITY OF NEVADA

NEW MEXICO STATE UNIVERSITY

OREGON STATE UNIVERSITY

UNIVERSITY OF OREGON

OSAKA UNIVERSITY

UNIVERSITY OF SOUTHERN CALIFORNIA
STANFORD UNIVERSITY

UNIVERSITY OF TOKYO

UNIVERSITY OF UTAH

WASHINGTON STATE UNIVERSITY

UNIVERSITY OF WASHINGTON

AMERICAN MATHEMATICAL SOCIETY CALIFORNIA RESEARCH CORPORATION SPACE TECHNOLOGY LABORATORIES NAVAL ORDNANCE TEST STATION 


\section{Pacific Journal of Mathematics}

\section{Vol. 14, No. 1 \\ May, 1964}

Richard Arens, Normal form for a Pfaffian .........................

Charles Vernon Coffman, Non-linear differential equations on cones in Banach

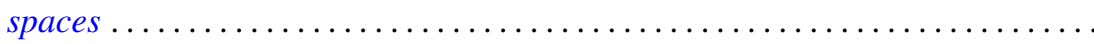

Ralph DeMarr, Order convergence in linear topological spaces ..............

Peter Larkin Duren, On the spectrum of a Toeplitz operator ................

Robert E. Edwards, Endomorphisms of function-spaces which leave stable all

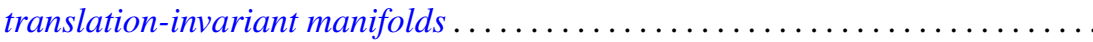

Erik Maurice Ellentuck, Infinite products of isols . . . . . . . . . . . . . . . . 49

William James Firey, Some applications of means of convex bodies . . . . . . . . 53

Haim Gaifman, Concerning measures on Boolean algebras ............. 61

Richard Carl Gilbert, Extremal spectral functions of a symmetric operator. . . . . . 75

Ronald Lewis Graham, On finite sums of reciprocals of distinct nth powers ..... 85

Hwa Suk Hahn, On the relative growth of differences of partition functions ...... 93

Isidore Isaac Hirschman, Jr., Extreme eigen values of Toeplitz forms associated

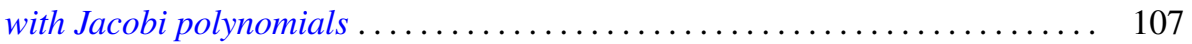

Chen-jung Hsu, Remarks on certain almost product spaces . . . . . . . . . . . 163

George Seth Innis, Jr., Some reproducing kernels for the unit disk . . . . . . . . . 177

Ronald Jacobowitz, Multiplicativity of the local Hilbert symbol . . . . . . . . . . . 187

Paul Joseph Kelly, On some mappings related to graphs ................. 191

William A. Kirk, On curvature of a metric space at a point . . . . . . . . . . . . 195

G. J. Kurowski, On the convergence of semi-discrete analytic functions . . . . . . . 199

Richard George Laatsch, Extensions of subadditive functions . . . . . . . . . . . 209

V. Marić, On some properties of solutions of $\Delta \psi+A\left(r^{2}\right) X \nabla \psi+C\left(r^{2}\right) \psi=0 \ldots 217$

William H. Mills, Polynomials with minimal value sets . . . . . . . . . . . 225

George James Minty, Jr., On the monotonicity of the gradient of a convex

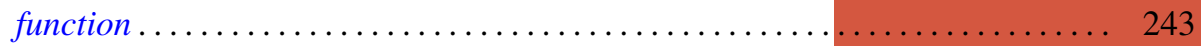

George James Minty, Jr., On the solvability of nonlinear functional equations of 'monotonic' type ................................... 249

J. B. Muskat, On the solvability of $x^{e} \equiv e(\bmod p) \ldots \ldots \ldots \ldots \ldots \ldots \ldots \ldots . \ldots \ldots$

Zeev Nehari, On an inequality of $P . R$. Bessack ................... 261

Raymond Moos Redheffer and Ernst Gabor Straus, Degenerate elliptic

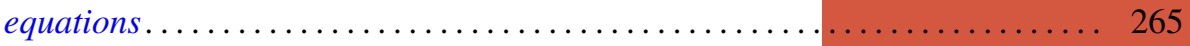

Abraham Robinson, On generalized limits and linear functionals . . . . . . . . . 269

Bernard W. Roos, On a class of singular second order differential equations with a

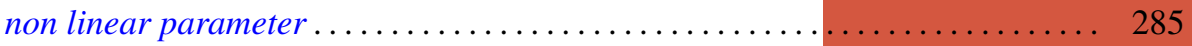

Tôru Saitô, Ordered completely regular semigroups . . . . . . . . . . . . . . . . 295

Edward Silverman, A problem of least area ....................... 309

Robert C. Sine, Spectral decomposition of a class of operators . . . . . . . . . 333

Jonathan Dean Swift, Chains and graphs of Ostrom planes . . . . . . . . . . . 353

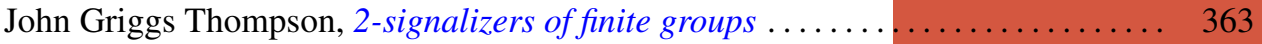

Harold Widom, On the spectrum of a Toeplitz operator . . . . . . . . . . . . . 365 